\title{
Are children just little adults when it comes to propofol injection pain?
}

\author{
Robin G. Cox MB BS MRCP(UK) FRCA FRCPC
}

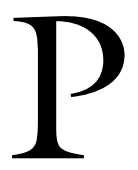

ROPOFOL is a deservedly popular anesthetic agent in pediatric practice, with the well-known benefits of smooth induction characteristics, antiemetic effect, rapid recovery and pleasant emergence. The drug appears to have a wide margin of safety and is less likely to cause significant hypotension than in the elderly population. With the exception of concerns that have been raised with its use for long-term sedation in the pediatric critical care environment, ${ }^{1}$ there appear to be few safety issues to unduly concern the anesthesiologist. Minor complications are a different issue. Most of these, such as excitatory events upon induction, are generally of a trivial nature, but the troublesome issue of pain on injection still remains and has never been consistently eradicated. ${ }^{2}$

Consider the following scenario. A happy little fellow arrives with doting parents for his hernia repair. A preoperative tour some days previously has helped reassure the family and the child is orientated to the hospital by child-life workers. On the day of surgery, local anesthetic cream is applied to the child's hand. The family meets with the anesthesiologist and it is planned to have a parent present for the induction. Everyone is happy and the anesthesiologist impresses everyone, including him/herself, by painlessly introducing the $i v$ catheter into the appropriate vein. When the propofol starts to run in, however, the child lets out a piercing scream and complains loudly about the "big owee!" in his arm. No one is that impressed any more. Will the child remember this event? Quite possibly. The parent will certainly remember.

Fortunately, this type of event is not that common, as most anesthesiologists administer lidocaine, either prior to the propofol with venous occlusion, or mixed with the propofol solution. There are some details of technique that seem to be important. Picard's large meta-analysis ${ }^{3}$ would suggest that lidocaine is most efficacious when given in advance of the propofol and by applying a tourniquet for up to $120 \mathrm{sec}$. Although a total of 6,246 patients were included in this analysis, most of the patients were adult. In practice, many children, particularly in the younger age group, object to a tourniquet being applied for this length of time. Whilst a circumferential squeeze with a pair of warm hands may be somewhat less threatening to the child, the reliability of this as a tourniquet may be questionable. Many pediatric anesthesiologists therefore add lidocaine to propofol and have good success with this mixture. Important points seem to be to add the lidocaine just prior to injection and to use an adequate amount of lidocaine. ${ }^{4}$ Morton ${ }^{5}$ has reported complete abolition of pain with a freshly prepared mixture of propofol $\left(3 \mathrm{mg} \cdot \mathrm{kg}^{-1}\right)$ and lidocaine $\left(1 \mathrm{mg} \cdot \mathrm{kg}^{-1}\right)$. This brief report was conducted in 50 unpremedicated children and the injections were made via a vein on the dorsum of the hand. The only concern with this mixture is that some children require higher doses of propofol $\left(4-5 \mathrm{mg} \cdot \mathrm{kg}^{-1}\right)$ for induction, particularly if used as a sole agent. When the induction dose is high, therefore, the lidocaine dose increases and toxicity becomes a concern.

Can other strategies reduce the pain on injection of propofol more than the appropriate use of lidocaine? Many approaches have been tried in the past, with mixed success. Two techniques are evaluated and presented in this month's Canadian Journal of Anesthesia. Rachel Pollard and colleagues, from B.C. Children's Hospital, Vancouver, have compared the effect of lidocaine and thiopental when added to propofol. ${ }^{6}$ The incidence of injection pain in the propofol/thiopental $(\mathrm{P} / \mathrm{T})$ group was $14 \%$, compared to $35 \%$ in the propofol/lidocaine $(\mathrm{P} / \mathrm{L})$ group. Some questions arise from this study. Firstly, it is not clear why the incidence of pain in the $\mathrm{P} / \mathrm{L}$ group was as high as $35 \%$. Morton, using the same dose of lidocaine

From the Division of Pediatric Anesthesia, Alberta Children's Hospital, University of Calgary, Calgary, Alberta, Canada.

Address correspondence to: Dr. Robin G. Cox, Division of Pediatric Anesthesia, Alberta Children's Hospital, 1820 Richmond Road S.W., Calgary, Alberta T2T 5C7, Canada. Phone: 403-943-7260; Fax: 403-943-7606; E-mail: robin.cox@calgaryhealthregion.ca 
$\left(1 \mathrm{mg} \cdot \mathrm{kg}^{-1}\right)$, found a zero incidence of pain with injection of propofol. ${ }^{5}$ Some of the difference may be due to the methods used to define injection pain. Morton provides little detail of the methods used to evaluate pain in his brief report and Pollard et al. use a non-validated pain evaluation tool. Another reason for a difference in these two reports is that, although the dose of lidocaine was the same, the concentration of lidocaine was actually lower in Pollard's study as she used an induction dose of $5 \mathrm{mg} \cdot \mathrm{kg}^{-1}$ of propofol compared with $3 \mathrm{mg} \cdot \mathrm{kg}^{-1}$ in Morton's patients. Thus it may well be that the crucial factor with lidocaine is the concentration rather than the total dose of lidocaine used.

Previous workers have studied the use of thiopental as a possible method of reducing propofol injection pain. Thiopental has been studied more often as pretreatment prior to propofol injection, sometimes in quite high doses. Cheng et al., for example, used a thiopental pretreatment dose of $3 \mathrm{mg} \cdot \mathrm{kg}^{-1}$ in children and found a $27 \%$ incidence of pain on injection with propofol. ${ }^{7}$ This was significantly lower than the comparison groups with lidocaine, although the doses of lidocaine used $\left(0.15,0.3\right.$ and $\left.0.6 \mathrm{mg} \cdot \mathrm{kg}^{-1}\right)$ were on the low side. It could be argued that a $3-\mathrm{mg} \cdot \mathrm{kg}^{-1}$ dose of thiopental is close to an induction dose on its own and that these children were virtually anesthetized prior to receiving propofol. Could not the same effect be produced by any other general anesthetic agent? A reasonable goal would be to avoid "paying a price" for reduced injection pain with other adverse effects, such as delayed recovery. In keeping with other authors, Pollard et al. do not provide any information as to the quality and time of recovery in their patients.

Is the routine use of thiopental justified in this context? The jury is still out on the question. There are theoretical objections to mixing two induction agents. Dosing errors may occur and the risk of allergic reactions, albeit very rare, increases slightly. The effects on recovery are unclear. Despite these objections, however, the technique does seem to be effective.

Another method to reduce propofol injection pain is described in this month's Canadian Journal of Anesthesia. Terence Beh and colleagues report a study from the Children's Hospital of Eastern Ontario, Ottawa, in which nitrous oxide inhalation was found to be effective in reducing propofol injection pain in children. ${ }^{8}$ The control group encountered a $36 \%$ incidence of propofol injection pain, which was reduced to $4 \%$ (one subject only) by inhalation of $50 \%$ nitrous oxide for two minutes. Several points are worth noting when reviewing this study. Firstly, both the control and treatment groups received an injection of propofol with a relatively low concentration of lidocaine added $(0.05 \%)$.
A concentration of lidocaine in this range might be expected to have a less than optimal effect, so it can be said that the control group was probably not the gold standard. Secondly, when injection pain did occur, even in the control group, this was generally scored as mild. Severe pain was not observed in either group. The third point to note is that the subjects, aged three to $12 \mathrm{yr}$, were required to breathe nitrous oxide for a full two minutes prior to propofol injection. An "effective seal" with the face mask was required for the study. Interestingly, none of the subjects were excluded because of failure to accept a mask for this period of time. In everyday pediatric practice, one has to wonder if the routine administration of nitrous oxide to reduce propofol injection pain is a practical option. If a child does tolerate the mask nicely, why not just turn on the sevoflurane as well? This would produce unconsciousness in less than two minutes.

Nitrous oxide has been used to alleviate the pain of a variety of procedures in children, including venous cannulation. ${ }^{9}$ Little has been written about its role in reducing propofol injection pain. A recently published article, however, in the French literature ${ }^{10}$ describes a study very similar to that of Beh et al. The treatment intervention in this study was also $50 \%$ nitrous oxide, however lidocaine was only added to propofol in the control group. This was therefore a comparison of nitrous oxide pretreatment $v$ s lidocaine. Interestingly, this study found no statistical difference in behavioural pain scoring between the two groups, although there were lower self-reported pain scores in the postoperative period pointing to a degree of amnesia in the nitrous oxide treated children. This study and that of Beh $e t a l$. would suggest that, even if nitrous oxide is used, it would also be appropriate to mix lidocaine with the propofol. As a final comment on nitrous oxide pretreatment, it should be recognized that this technique would not be appropriate for a rapid sequence induction, where preoxygenation with $100 \%$ oxygen is recommended.

What role might these two techniques, thiopental admixture and nitrous oxide pretreatment, play in reducing propofol injection pain in children? Although superficially effective, probably neither method is the perfect solution to the problem. There are unanswered questions about the recovery profile after thiopental/propofol admixture and the option of pretreatment with nitrous oxide may not always be practical or appropriate. For many pediatric anesthesiologists, an adequate dose of lidocaine mixed with the propofol will likely remain the standard method of reducing propofol injection pain in children, at least until something better comes along. 
Les enfants ne sont-ils que de jeunes adultes face à la douleur causée par l'injection de propofol ?

Le propofol est à juste titre un anesthésique populaire en pratique pédiatrique étant donné les avantages bien connus d'induction en douceur, d'effet antiémétique, de récupération rapide et de retour à la conscience agréable. Le médicament semble avoir une grande marge de sécurité et causer moins d'hypotension importante chez les gens âgés. Si on exclut les questions soulevées sur son usage comme sédatif de long terme en soins intensifs pédiatriques, ${ }^{1}$ peu de problèmes d'innocuité ne devraient vraiment préoccuper les anesthésiologistes. Les complications mineures, c'est autre chose. La plupart d'entre elles, comme une surexcitation provoquée à l'induction, sont généralement sans importance, mais la question troublante de la douleur lors de l'injection demeure encore et n'a pu être éliminée. ${ }^{2}$

Envisageons l'exemple suivant. Un joyeux petit bonhomme se présente, accompagné de ses parents qui l'adorent, pour subir la réparation d'une hernie. Une visite préopératoire des lieux, quelques jours avant, a permis de rassurer la famille et l'enfant s'est familiarisé à l'environnement hospitalier grâce au personnel attentionné. Le jour de l'opération, une crème anesthésique locale est appliquée sur la main de l'enfant. La famille rencontre l'anesthésiologiste et on prévoit la présence d'un parent pendant l'induction de l'anesthésie. Tout le monde est heureux et l'anesthésiologiste surprend chacun, y compris lui-même, en introduisant le cathéter iv dans la bonne veine sans provoquer de douleur. Cependant, quand le propofol commence à circuler dans l'appareil, l'enfant se met à hurler et à se plaindre bruyamment du "gros bobo" à son bras. Plus personne n'est encore impressionné. L'enfant se souviendra-t-il de cet événement ? Fort possiblement. Le parent, lui, ne l'oubliera certainement pas.

Heureusement, ce genre d'événement n'est pas très fréquent, puisque la plupart des anesthésiologistes administrent de la lidocaïne, soit avant le propofol lors de l'occlusion veineuse, soit mélangé à la solution de propofol. Certains détails techniques semblent impor- tants. La méta-analyse de Picard ${ }^{3}$ montre que la lidocaïne est plus efficace quand on l'administre avant le propofol en présence d'un garrot appliqué pendant au plus 120 s. Même si 6246 patients ont été choisis pour cette analyse, la majorité était des adultes. En pratique, de nombreux enfants, surtout chez les plus jeunes, refusent de subir un garrot pendant ce laps de temps. Quoiqu'une compression circulaire faite par une paire de mains chaudes puisse être moins menaçante pour l'enfant, la fiabilité de ce garrot est discutable. Nombre d'anesthésiologistes pédiatriques ajoutent donc avec succès de la lidocaïne au propofol. Le plus important semble d'ajouter la lidocaïne juste avant l'injection et d'en utiliser une quantité appropriée. ${ }^{4}$ Morton ${ }^{5}$ a constaté l'abolition complète de la douleur avec un mélange frais de propofol $\left(3 \mathrm{mg} \cdot \mathrm{kg}^{-1}\right)$ et de lidocaïne $\left(1 \mathrm{mg} \cdot \mathrm{kg}^{-1}\right)$. Ce rapport sommaire résume l'expérience menée auprès de 50 enfants qui n'ont pas reçu de prémédication et qui ont reçu une injection dans une veine du dos de la main. Le seul problème de ce mélange c'est qu'il faut augmenter la dose de propofol à l'induction pour certains enfants (4-5 $\left.\mathrm{mg} \cdot \mathrm{kg}^{-1}\right)$, surtout si le propofol est utilisé seul. En conséquence, quand la dose d'induction est élevée, la dose de lidocaïne augmente et on commence à craindre la toxicité.

Peut-on réduire autrement qu'avec de la lidocaïne la douleur à l'injection de propofol ? De nombreuses approches ont connu des succès mitigés. Deux techniques sont évaluées dans le présent numéro du Journal canadien d'anesthésie. Rachel Pollard et ses collègues, du B.C. Children's Hospital, à Vancouver, ont comparé l'effet de la lidocaïne et du thiopental ajouté au propofol. ${ }^{6}$ L'incidence de douleur d'injection a été de $14 \%$ chez les patients du groupe propofol/thiopental $(\mathrm{P} / \mathrm{T})$ et de $\mathbf{3 5} \%$ chez ceux du groupe propofol/lidocaïne $(\mathrm{P} / \mathrm{L})$. Ces résultats soulèvent des questions. D'abord, pourquoi l'incidence de douleur a-t-elle été aussi élevée que $35 \%$ dans le groupe $\mathrm{P} / \mathrm{L}$ ? En effet, Morton, qui a utilisé la même dose de lidocaïne $\left(1 \mathrm{mg} \cdot \mathrm{kg}^{-1}\right)$, n'a connu aucune incidence de douleur avec l'injection de propofol. ${ }^{5}$ Une partie de l'écart vient sans doute des méthodes utilisées pour définir la douleur à l'injection. Morton ne fournit que peu de détails sur les méthodes utilisées pour évaluer la douleur dans son rapport sommaire et Pollard et coll. se servent d'un outil d'évaluation non validé. Une autre raison de la différence entre ces deux rapports est que, même si la dose de lidocaïne est la même, la concentration du médicament est en fait plus faible dans l'étude de Pollard, car elle a utilisé $5 \mathrm{mg} \cdot \mathrm{kg}^{-1} \mathrm{de}$ propofol comparé à $3 \mathrm{mg} \cdot \mathrm{kg}^{-1}$ dans l'étude de Morton. Donc, il se pourrait que le facteur détermi- 
nant soit la concentration de lidocaïne plutôt que la dose utilisée.

Des chercheurs avant eux ont étudié l'usage du thiopental pour réduire la douleur à l'injection du propofol. Le thiopental a été étudié plus souvent comme prémédication à l'injection de propofol, parfois avec des doses assez élevées. Cheng et coll., par exemple, ont utilisé une dose de prémédication de 3 $\mathrm{mg} \cdot \mathrm{kg}^{-1}$ de thiopental chez des enfants et trouvé une incidence de $27 \%$ de douleur à l'injection de propofol. ${ }^{7}$ Ce résultat était significativement plus bas qu'avec la lidocaïne, même si les doses de lidocaïne utilisées $\left(0,15,0,3\right.$ et $\left.0,6 \mathrm{mg} \cdot \mathrm{kg}^{-1}\right)$ étaient faibles. Mais on pourrait dire que la dose de $3-\mathrm{mg} \cdot \mathrm{kg}^{-1}$ de thiopental est pratiquement une dose d'induction en elle-même et que ces enfants ont été virtuellement anesthésiés avant de recevoir le propofol. Le même effet peut-il se produire avec tout autre anesthésique général ? Il peut être raisonnable de vouloir éviter de "payer le prix" pour réduire la douleur à l'injection, malgré d'autres effets secondaires comme un réveil retardé. Comme d'autres auteurs, Pollard et coll. ne donnent aucune information sur la qualité et le temps du réveil de leurs patients.

Est-ce que l'usage courant de thiopental est justifié dans ce contexte ? Les experts discutent toujours de la question. Il y a des objections théoriques au mélange de deux agents d'induction. Des erreurs de dosage peuvent se produire et le risque de réactions allergiques, quoique très rare, peut augmenter légèrement. Les effets sur le réveil ne sont pas clairs. En dépit de ces objections, toutefois, la technique semble efficace.

Une autre méthode de réduction de la douleur à l'injection de propofol est décrite dans le présent numéro. Terence Beh et coll. mentionnent une étude réalisée au Children's Hospital of Eastern Ontario, à Ottawa, dans laquelle du protoxyde d'azote s'est montré efficace chez des enfants. ${ }^{8}$ Les témoins ont connu une incidence de $36 \%$ de douleur, réduite à 4 $\%$ (un sujet seulement) par l'inhalation de protoxyde d'azote à $50 \%$ pendant deux minutes. Certains aspects de cette étude sont à souligner. Premièrement, les patients des groupes témoin et expérimental ont reçu une injection de propofol avec l'ajout d'une concentration relativement faible de lidocaïne $(0,05 \%)$. On peut prévoir qu'une telle concentration de lidocaïne aura un effet moins qu'optimal. Ce qui amène à dire que le groupe témoin n'était probablement pas une bonne référence. Deuxièmement, la douleur à l'injection était modérée, même dans le groupe témoin. Aucune douleur sévère n'a été enregistrée dans les deux groupes. Troisièmement, les sujets, de 3 à 12 ans, devaient inhaler du protoxyde d'azote pendant deux minutes avant l'injection de propofol. Une étanchéité efficace du masque a été nécessaire pour l'étude. Il faut noter qu'aucun sujet n'a été exclu parce qu'il a refusé le masque pendant cette période de temps. Dans la pratique pédiatrique quotidienne, on peut se demander si l'administration routinière de protoxyde d'azote pour réduire la douleur à l'injection de propofol constitue une option pratique. Si un enfant tolère bien le masque, pourquoi ne pas administrer tout simplement du sévoflurane ? Cela provoquera l'inconscience en moins de deux minutes.

Le protoxyde d'azote a été utilisé pour soulager la douleur pendant de nombreuses interventions chirurgicales chez les enfants, y compris la canulation veineuse. 9 On a peu écrit sur son rôle de réducteur de la douleur à l'injection de propofol. Un article récemment publié dans une revue française ${ }^{10}$ décrit cependant une étude très semblable à celle de Beh et coll. L'expérience comportait également du protoxyde d'azote à $50 \%$, mais seulement de la lidocaine était ajoutée au propofol chez les patients témoins. C'était donc la comparaison d'une prémédication avec le protoxyde d'azote $v s$ la lidocaïne. Fait intéressant, cette étude n'a pas trouvé de différence statistique intergroupe des scores de douleur, même s'il y a eu des scores de douleur postopératoire indiquant un certain degré d'amnésie chez les enfants traités avec le protoxyde d'azote. Cette étude et celle de Beh et coll. montrent que, même avec l'usage de protoxyde d'azote, il pourrait être approprié de mélanger de la lidocaïne au propofol. Finalement, on doit reconnaitre que la prémédication avec le protoxyde d'azote ne convient pas à une séquence d'induction rapide quand la préoxygénation avec $100 \%$ d'oxygène est recommandée.

Quel rôle ces deux techniques, l'ajout de thiopental et la prémédication avec le protoxyde d'azote, jouent-elles dans la réduction de la douleur à l'injection de propofol chez les enfants ? Bien que superficiellement efficace, aucune des méthodes ne représente probablement la solution parfaite au problème. Certaines questions concernant le profil de récupération après l'administration d'un mélange de thiopental et de propofol sont sans réponse. De même, la possibilité d'une prémédication avec du protoxyde d'azote ne représente pas un choix toujours pratique ou approprié. Pour de nombreux anesthésiologistes pédiatriques, une dose suffisante de lidocaïne ajouté au propofol demeure la meilleure méthode pour réduire la douleur à l'injection de propofol chez les enfants, en attendant mieux.

\section{References}

1 Parke TJ, Stevens JE, Rice AS, et al. Metabolic acidosis and fatal myocardial failure after propofol infusion in 
children: five case reports. BMJ 1992; 305: 613-6.

2 Smith I, White PF, Nathanson M, Gouldson R.

Propofol. An update on its clinical use. Anesthesiology 1994; 81: 1005-43.

3 Picard P, Tramer MR. Prevention of pain on injection with propofol: a quantitative systematic review. Anesth Analg 2000; 90: 963-9.

4 Cameron E, Johnston G, Crofts S, Morton NS. The minimum effective dose of lignocaine to prevent injection pain due to propofol in children. Anaesthesia 1992; 47: 604-6.

5 Morton NS. Abolition of injection pain due to propofol in children. Anaesthesia 1990; 45: 70.

6 Pollard RC, Makky S, McFadzean J, Ainsworth L, Goobie SM, Montgomery CJ. An admixture of $3 \mathrm{mg} \cdot \mathrm{kg}^{-1}$ of propofol and $3 \mathrm{mg} \cdot \mathrm{kg}^{-1}$ of thiopentone reduces pain on injection in pediatric anesthesia. Can J Anesth 2002; 49: 1064-69.

7 Cheng KI, Tang CS, Chiu SL, et al. Injection pain with propofol: the effectiveness of thiopentone on induction. Kaohsiung J Med Sci 1998; 14: 480-5.

8 Beh T, Splinter W, Kim J. In children nitrous oxide decreases the pain of injection of propofol mixed with lidocaine. Can J Anesth 2002; 49: 1061-63.

9 Henderson JM, Spence DG, Komocar LM, Bonn GE, Stenstrom RJ. Administration of nitrous oxide to pediatric patients provides analgesia for venous cannulation. Anesthesiology 1990; 72: 269-71.

10 Lembert N, Wodey E, Geslot D, Ecoffey C. Prévention de la douleur à l'injection du propofol chez l'enfant: comparaison de l'inhalation de protoxyde d'azote à l'injection de lidocaine (French). Ann Fr Anesth Reanim 2002; $21: 263-70$. 